\title{
Modelling fluctuations of financial time series: from cascade process to stochastic volatility model
}

\author{
J.F. Muzy ${ }^{1}$, J. Delour ${ }^{1}$ and E. Bacry ${ }^{2}$ \\ ${ }^{1}$ Centre de Recherche Paul Pascal, Avenue Schweitzer 33600 Pessac, France \\ ${ }^{2}$ Centre de Mathématiques appliquées, Ecole Polytechnique, 91128 Palaiseau Cedex, France
}

November 5, 2018

\begin{abstract}
In this paper, we provide a simple, "generic" interpretation of multifractal scaling laws and multiplicative cascade process paradigms in terms of volatility correlations. We show that in this context 1 /f power spectra, as recently observed in Ref. [20], naturally emerge. We then propose a simple solvable "stochastic volatility" model for return fluctuations. This model is able to reproduce most of recent empirical findings concerning financial time series: no correlation between price variations, long-range volatility correlations and multifractal statistics. Moreover, its extension to a multivariate context, in order to model portfolio behavior, is very natural. Comparisons to real data and other models proposed elsewhere are provided.
\end{abstract}

\section{Introduction}

As shown by most recent empirical studies on huge amount of data, the market price changes are characterized by several "universal" features [1, 2]: price increments are not correlated, volatilities are strongly (power-law) correlated and price increment probability density function (pdf) shapes depend on the time scale. From quasi Gaussian at rather large time scales, these pdf are characterized by fat tails at fine scales. Many authors in the recently emerged field of "econophysics" 11, 2, 3] as well as in classical empirical finance, aim at proposing simple, discrete or continuous time models that are able to account for these observations. Among all the proposed models, one can distinguish several streams, from the simplest Brownian process, that constitutes the main tool used by practitians, to the class of "heteroskedastic" nonlinear processes as proposed in Refs. [6, 7]. To account for the letpokurtic nature of the small scale pdf, Mandelbrot [4] and Fama [- proposed the Levy stable paradigm that has been recently improved in the "truncated Levy" version [8, 1, 2]. More recently, an interesting comparison between market price variations and the fluctuations of the fluid velocity field in fully developed turbulence has been suggested [9]. Besides the real pertinence of such an analogy that has been widely commented [11, 12, 2], this work opens the door to another important paradigm to model

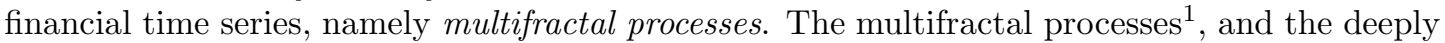
connected mathematics of large deviations and multiplicative cascades, are well known to be useful to describe the intermittent nature of fully developed turbulence [21]. Recent empirical

\footnotetext{
${ }^{1}$ people sometimes refer to "multi-affine" processes or processes that display "multi-scaling"
} 
findings 10, 13, 15, 16] suggest that in finance, this framework is also likely to be pertinent as far as the time scale dependence of the statistical properties of price variations is concerned.

Our purpose in this paper is twofold. First, we make a brief review of multifractals in order to specify what is a multifractal process. We try to provide several complementary points of view and to understand what are the main ingredients for "multi-scaling". We also comment about the criticisms raised by several authors about multifractality in finance. Our second goal is to propose a simple multifractal "stochastic volatility" model that captures very well all the above mentionned features of financial fluctuations. This model, that has been originally introduced in Ref. [18], is compared to real data and some models proposed elsewhere. We discuss its possible multivariate extension in order to use it in management applications. The paper is organized as follows. The review on multifractal processes is made in section 2. We introduce notations, the related notions of multi-scaling, scale-invariance, cascade process and self-similarity kernel. We illustrate our purpose using empirical estimates for some high frequency financial data. In section 3 we review some findings of Ref. [10] concerning the magnitude correlations for cascade models and suggest a link with $1 / f$ processes as recently observed in financial time series. In section 4 we introduce the multifractal random walk defined in Ref. 18 as a stochastic volatility model. We discuss its main properties and propose a natural multivariate generalization. Our discussion is illustrated by numerical simulations. In section 5 we propose estimators for the few parameters of our model and compute them for some intraday and daily time series. In section 6 we discuss some related works about multifractality in finance. Conclusions and some prospects are reported in section 7 .

\section{Multifractal processes and cascade models}

In this section we briefly discuss the related notions of multifractality and multiplicative cascade. Most of the ideas and concepts that we recall below have been introduced in the field of fully developed turbulence where people aim at accounting for the so-called "intermittency phenomenon" (for a review of this subject see e.g., 21]).

\subsection{Multifractality of financial time series}

Let us consider the variations of a stochastic process $X(t)$ at a time scale $l$. For that purpose, one can consider the increments of the process, $\delta_{l} X(t)=X(t+l)-X(t)$ or more generally its wavelet transform 22, 23, 24]

$$
T(t, l)=l^{-1} \int \psi\left(\frac{t^{\prime}-t}{l}\right) X\left(t^{\prime}\right) d t^{\prime}
$$

where $\psi(t)$ is the so-called analyzing wavelet, i.e, a function well localized in both Fourier and direct spaces . Let us denote $M(q, l)$ the order $q$ absolute moment of $\delta_{l} B(t)$ or $T(t, l)$, (in this paper $E($.$) will be used for the mathematical expectation and we will always suppose that the$ considered processes has stationary increments)

$$
M(q, l)=E\left(\left|\delta_{l} X(t)\right|^{q}\right) .
$$

We will say that the process is scale-invariant, if the scale behavior of the absolute moment $M(q, l)$ is a power law. Let us call $\zeta_{q}$ the exponent of this power law, i.e.,

$$
M(q, l) \sim C_{q} l^{\zeta_{q}}
$$

\footnotetext{
${ }^{2}$ One nice property of wavelet transform is that it can be inverted, i.e., one can recover the original signal from its wavelet coefficients. Another interesting feature is that there exist orthonormal wavelet bases. Such bases are very useful for signal synthesis and modelling, as it is illustrated for cascade processes in Ref. [33]
} 

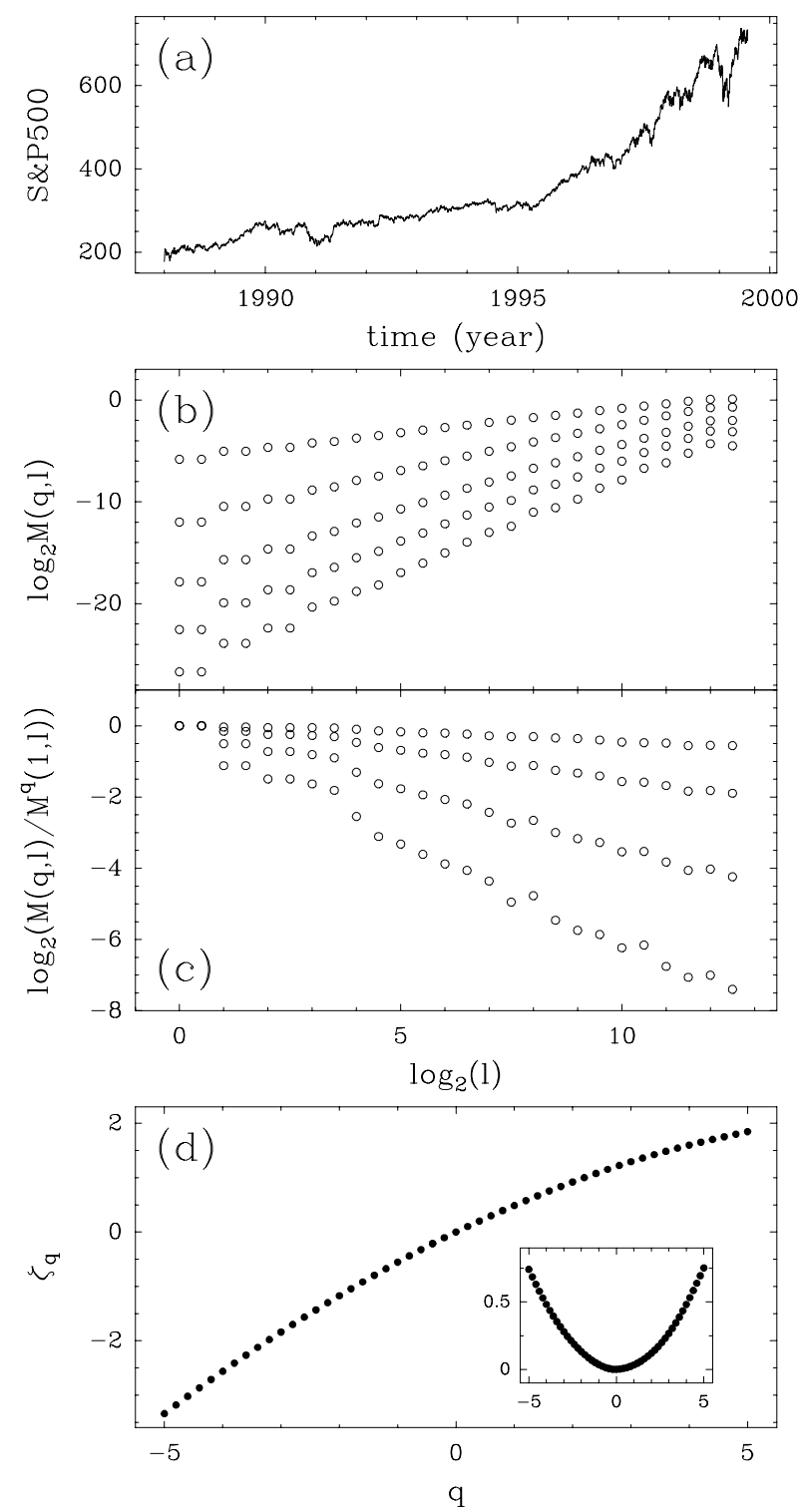

Figure 1: Multifractal Analysis of the intraday future S\&P500 index over the period 1988-1999. (a) Plot of the original index time-series. The analyzed time-series is the detrended and de-seasonalized logarithm of this series. (b) Log-log plots of $M(q, l)$ versus $l$ for $q=1,2,3,4,5$. The time scales $l$ range from 10 minutes to 1 year. (c) $\log _{2}\left(M(q, l) / M(1, l)^{q}\right)$ for $q=2,3,4,5$. Such plots should be horizontal for a process that is not multifractal. (d) $\zeta_{q}$ spectrum for the S\&P 500 fluctuations. The plot in the inset is the parabolic nonlinear part of $\zeta_{q}$. 
where $C_{q}$ is a prefactor that will be interpreted below. The process is called monofractal if $\zeta_{q}$ is a linear fonction of $q$ and multifractal if $\zeta_{q}$ is nonlinear. Note that, from the concavity of the moments of a random variable, it is easy to show that $\zeta_{q}$, as defined from the scaling behavior (2) in the limit $l \rightarrow 0^{+}$, is necessarily a convex fonction of $q$. The same argument leads to the conclusion that such scaling behavior with a nonlinear $\zeta_{q}$ cannot hold for all scales $l$. Thus, for a multifractal process there exists at least one characteristic time $T$ (hereafter referred to as the integral time) above which the behavior (2) is no longer valid. Multifractality has been introduced in the context of fully developed turbulence in order to describe the spatial fluctuations of the fluid velocity at very high Reynolds number [21]. As suggested by recent studies [9, 10, 13, 15, 16], multifractality is likely to be a pertinent concept to account for the prices fluctuations in financial time-series. This is illustrated in Fig. 1 where the $\zeta_{q}$ function is estimated for the future S\&P500 index over the period 1988-1999. The original intraday time-series has been sampled at a $10 \mathrm{mn}$ rate (Fig. 1(a)) in order to obtain equi-sampled data. We consider the associated continuously compounded return time-series, (i.e., the logarithm of the index value) that has been detrended and de-seasonalized. The $\zeta_{q}$ spectrum in Fig. If (c) is obtained using linear regression fit of "log-log" representations of the behavior of the $q$-th order moment versus the time scale as illustrated in Fig. 1 1 (b). In this figure, the scales span an interval from 10 minutes to approximately 1 year. Moment estimates at larger time scales are very poor because of the finite size of the overall record. From the linear behavior of such curves, one clearly sees that the scale-invariance hypothesis is satisfied over around 3 decades. In Fig. 1(b) we have plotted $\log _{2} \frac{M(q, l)}{M(1, l)^{q}}$ versus $\log _{2}(l)$. The fact that such plots are not constant reflects the nonlinearity the $\zeta_{q}$ spectrum. The future S\&P500 can thus be considered, at least at this description level, as a multifractal signal. Let us notice that we have computed, in Fig. 1(d), the $\zeta_{q}$ values for $q$-th order moments that include negative values of $q$. This can be achieved using a wavelet based technique that has been introduced in Refs. [25, 26, 27, 28]. This spectrum turns out to be well fitted by a parabolic shape $\zeta_{q}=0.53 q-0.015 q^{2}$. The non linear parabolic component of $\zeta_{q}$ has been plotted in the inset of Fig. 1 1 (d).

\subsection{Multifractal processes, self-similar processes and multiplicative cascades.}

Multifractality (in the sense defined above) is a notion that is often related to an underlying multiplicative cascading process. In the context of deterministic functions the situation is rather clear since the analyticity of the $\zeta_{q}$ spectrum is deeply connected to the self-similarity properties of the function [26, 27, 28, 29]. Roughly speaking, a function is self-similar if it can be written as a multiplicative cascade in an appropriate space-scale (or time-scale) representation [27, 26, 29]. In that context, the so-called multifractal formalism is valid, i.e., one can relate the $\zeta_{q}$ spectrum to the $D(h)$ singularity spectrum that provides information about the statistical distribution of singularity (Hölder) exponents $h$. The things are somehow more complex for stochastic processes. One of the goals of this paper is to provide some simple elements about this subject.

In the mathematical literature, a process $X(t)$ is called self-similar of exponent $H$ if $\forall \lambda>0$, $\lambda^{-H} X(\lambda t)$ is the same process as $X(t)$. According to this definition, the Brownian motion is self-similar with an exponent $H=1 / 2$. This definition is however too restrictive for our purpose since it excludes multifractal processes. Indeed, let us consider $P_{l}(\delta X)$ the probability density function (pdf) of $\delta_{l} X(t)$. If $X(t)$ is self-similar with an exponent $H$, then it is easy to prove

\footnotetext{
${ }^{3}$ The amplitude of the return variations in each intraday period is normalized according to the estimated U-shaped intraday r.m.s.

${ }^{4}$ Note that from stationarity of the increments, the law of $\delta_{l} X(t)$ is the same as the law of $X(l)$ if one assumes that $X(0)=0$.
} 
that

$$
P_{l}(\delta X)=\lambda^{H} P_{\lambda l}\left(\lambda^{H} \delta X\right) .
$$

Then, the moments at scale $l$ and $L=\lambda l$ are related by

$$
M(q, l)=C_{q}\left(\frac{l}{L}\right)^{q H},
$$

with $C_{q}=M(q, L)$. Thus one has a "monofractal" process with $\zeta_{q}=q H$. In order to account for multifractality, one has to generalize this classical definition of self-similarity. This can be done by introducing a weaker notion, as originally proposed in the field of fully developed turbulence by B. Castaing and co-authors [31]. According to Castaing's definition of self-similarity, a process is self-similar if the increment pdf's at scales $l$ and $L=\lambda l(\lambda>1)$ are related by the relationship 31, 32:

$$
P_{l}(\delta X)=\int G_{l, L}(u) e^{-u} P_{L}\left(e^{-u} \delta X\right) d u,
$$

where the self-similarity kernel $G_{l, L}$ depends only on $l / L$. Let us note that this definition generalizes Eq. (3) that corresponds to the "trivial" case $G_{l, L}(u)=\delta(u-H \ln (l / L))$. This equation basically states that the pdf $P_{l}$ can be obtained through a "geometrical convolution" between the kernel $G_{l, L}$ and the pdf $P_{L}$. A simple argument shows that the logarithm of the Fourier transform of the kernel $G_{l, L}$ can be written as $F_{l, L}(k)=\ln \hat{G}_{l, L}(k)=F(k) \ln (l / L) 5$. Thus, from Eq. (5), one can easily show that the $q$ order absolute moments at scales $l$ and $L$ are related by:

$$
M(q, l)=\hat{G}_{l, L}(-i q) M(q, L)=M(q, L)\left(\frac{l}{L}\right)^{F(-i q)},
$$

and then $C_{q}=M(q, L)$ and $\zeta_{q}=F(-i q)$. A nonlinear $\zeta_{q}$ spectrum implies that $F$ is nonlinear and thus that $G$ is different from a Dirac delta function $[$. For example, the simplest non linear case is the so-called log-normal model that corresponds to a parabolic $\zeta_{q}$ function and thus to a function $G$ that is Gaussian.

The equation (5) can be interpreted as follows: the pdf at scale $l, P_{l}$ is written as a weighted superposition of the rescaled versions of the pdf at scale $L, P_{L}$, the self-similarity kernel $G_{l, L}$ being the associated distribution of weights. In the case of a monofractal process as described by Eq. (3), a single value of $u$ is sufficient in the equation (5) since $P_{l}$ and $P_{L}$ have the same shape and differ only by the scale factor $e^{-u}=(l / L)^{H}=\lambda^{H}$. This explains the Dirac function for the kernel $G$. This situation can be easily generalized by considering other shapes for the kernel $G_{l, L}$. In that case, the shapes of the pdf $P_{l}$ across scales are no longer the same: when going to small scales, fat tails emerge and the pdf become strongly leptokurtic (see Refs. [9, 31] or Fig. (1).

Let us now make the link with multiplicative cascades. This can be easily done if one consider discrete scales $l_{n}=2^{-n} L$. Let us suppose that the local variation of the process $\delta_{l_{n}} X$ at scale $l_{n}$ is obtained from the variation at scale $L$ as

$$
\delta_{l_{n}} X(t)=\left(\prod_{i=1}^{n} W_{i}\right) \delta_{L} X(t)
$$

where $W_{i}$ are i.i.d. random positive factors. This is the cascade paradigm. Realizations of such processes can be constructed using orthonormal wavelet bases as discussed in Ref. [33]. If one

\footnotetext{
${ }^{5}$ It essentially results from the fact that $G_{l, L}$ depends only on $l / L$ and satisfies the semi-group composition law $G_{l_{1}, l_{3}}=G_{l_{1}, l_{2}} * G_{l_{2}, l_{3}}$ where $l_{1} \leq l_{2} \leq l_{3}$ and $*$ is the convolution product [31, 32].

${ }^{6}$ Note that from the above mentionned semi-group property, the Levy theorem 30 implies that $G$ is necessarily an infinitely divisible law
} 
defines the magnitude $\omega(t, l)$ at time $t$ and scale $l$ as the logarithm of "local volatility" [10]:

$$
\omega(t, l)=\frac{1}{2} \ln \left(\left|\delta_{l} X(t)\right|^{2}\right),
$$

then the previous cascade equation becomes a simple random walk equation, at fixed time $t$, versus the logarithm of scales:

$$
\omega\left(t, l_{n+1}\right)=\omega\left(t, l_{n}\right)+\ln \left(W_{n+1}\right) .
$$

If the noise $\ln W_{i}$ is normal $N\left(\mu, \lambda^{2}\right)$, the pdf of $\omega, P_{l}(\omega)$, thus satisfies a simple diffusion equation with a Gaussian kernel:

$$
P_{l_{n}}(\omega)=\left(N\left(\mu, \lambda^{2}\right)^{* n} * p_{L}\right)(\omega)
$$

where $*$ is the convolution product. Going back to the original variable $\delta X$, the previous equation corresponds exactly to Castaing's formulation of self-similarity (5) with the log-normal propagator:

$$
G_{l_{n}, L}=N\left(\mu, \lambda^{2}\right)^{* n}=N\left(n \mu, n \lambda^{2}\right) .
$$

Conversely, let us consider a process that satisfies Castaing's equation with a normal kernel $G$. This means that one can write,

$$
\delta_{l} X(t) \equiv W \delta_{2 l} X(t)
$$

where $\equiv$ means the equality in law of the two random variables and $W$ is a log-normal random variable which mean $\mu$ and variance $\lambda^{2}$ do not depend on $l$. By iterating this equation $n$ times, one thus recover, at least heuristically, the cascade equation (77). Thus, the cascade picture across scales, constitutes a kind of paradigm of non-trivial self-similar processes. As explained in Ref. [18], the problem with such processes is that they involve representations (e.g., orthonormal wavelet bases) that are constructed on a discrete set of scales (e.g., dyadic scales $l_{n}=2^{-n}$ ) and in turn cannot be invariant under continuous scale dilations.

\section{Magnitude correlations and 1/f spectra}

We have seen in the previous section that multifractality can be interpreted as a diffusion of the magnitude of the variations of the return from large time scales to small time scales. In the financial framework, magnitudes at all scales are nothing but a logarithmic representation of local volatilities. In this section we would like to address the problem of volatility correlations. The "heteroskedastic" nature of financial time-series is now a well established empirical fact. Volatility possesses long-range positive correlations: periods of strong activity alternate with quiet periods. A lot of models have been proposed to account for this phenomenon from the famous GARCH models to various stochastic volatility models. Let us proceed with the multifractal and cascade picture and study what kind of correlations are associated to these models. This problem has already been considered by Arneodo, Muzy and Sornette in Ref. 10] (see also Refs [33, 34]). These authors have shown that a log-normal cascade model on the dyadic tree associated to the orthonormal wavelet representation leads naturally to magnitude correlation functions $C_{\omega}(l, \tau)=\operatorname{Cov}(\omega(t, \tau), \omega(t+l, \tau))$ that behave as $-\lambda^{2} \ln (l / T)$ for $T>l>\tau$. This behavior has been shown to provide good fits of the empirical estimates of the correlation functions from real data $[10]$. In Fig. 2 2 is reported the magnitude correlation function $C_{\omega}(l, \tau)$ (we choose $\tau=10 \mathrm{~min}$ ) of the S\&P500 time series studied in Fig. I. One can see that, when plotted versus the logarithm of the time lag, $\ln (l)$, the correlation function decreases linearly with a slope $\lambda^{2} \simeq 0.025$. The intercept of such straight line provides an estimator of the integral time $T$ that is, in our case, approximately $T \simeq 3$ years (note that because we get an estimate 

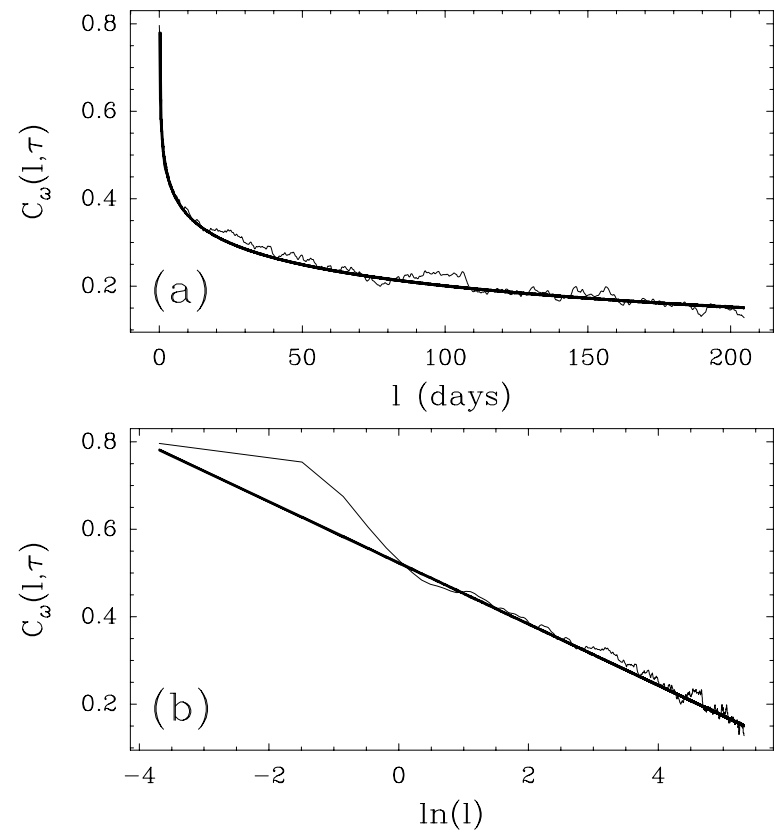

Figure 2: Magnitude correlation function of the $\mathbf{S} \& \mathbf{P} \mathbf{5 0 0}$ future. (a) $C_{\omega}(l, \tau)$ versus $l$ for $\tau=10 \mathrm{~min}$. The solid line represents a fit according to the cascade model logarithmic expression. (b) $C_{\omega}(l, \tau)$ versus $\ln (l)$. The cascade model predicts a linear behavior that crosses the y-axis at $\ln (l)=\ln (T)$. The small scale cross-over is due to the smoothing window used to estimate the local magnitude $\omega(t, \tau)$. 
of $\ln (T)$ the error on the value of $T$ is very large). We have checked that those results are stable when changing the reference time $\tau$ for return calculation. As it will be illustrated in section 5 , for various financial time series, the "cascade ansatz" is very pertinent to describe the volatility correlations. Let us notice that the very slow (logarithmic) decrease of the correlation functions for time lags below the integral time $T$, is reminiscent of the ultrametric nature of the tree naturally associated to the time-scale (or time-frequency) representation [10, 33, 34]. Moreover, let us remark that, as far as power spectrum is concerned, Gaussian processes with such correlation functions can be seen as " $1 / f$ " processes. Indeed, if the correlation function is given by the above expression, the power spectrum can be shown to reduce to

$$
S(f)=2 \lambda^{2} f^{-1} \int_{0}^{T f} x^{-1} \sin (x) d x .
$$

In the high frequency limit $f \rightarrow+\infty$, we then have $S(f) \sim \lambda^{2} \pi f^{-1}$. Another intuitive way to understand this property, comes from the fact that the logarithmic decay of the correlation function can be understood as the limit $H \rightarrow 0$ in the power-law correlation function $k^{-2 H}$ of a fractional gaussian noise of exponent $H$. This property will be explicitely used in the discussion of section 6.3. Let us finally remark that $1 / f$ spectra have been observed in a wide range of applications [35]. Recently, Bonanno et al. [20] suggested the possible pertinence of such processes to account for the fluctuations of the number of trades of different stocks.

\section{A simple solvable multifractal model}

As emphasized previously, multiplicative cascade models represent the paradigm of multifractal processes in that they contain the main ingredient leading to multifractality, i.e, the scale evolution of the magnitudes, from coarse to fine scales, is a random walk. Besides the problems of continuous scale invariance and stationarity of standard hierarchical constructions of such processes, they cannot be formulated using a stochastic evolution equation as one would expect for a model for financial time series. In this section we propose a "stochastic volatility" model that has been introduced in Ref. [18], that does not possess any of these drawbacks: it has stationary increments, it has log-normal multifractal properties and is invariant under continuous dilations. The key idea underlying this model is that the stochastic volatility possesses, as for cascading processes, a " $1 / f$ " spectrum, or, more precisely, a correlation function with a logarithmic behavior.

\subsection{The multifractal random walk}

Let us briefly recall the construction of the multifractal random walk (MRW) proposed in 18]. A discretized version of the model $X_{\Delta t}$ (using a time discretization step $\Delta t$ ) is built by adding up $t / \Delta t$ random variables :

$$
X_{\Delta t}(t)=\sum_{k=1}^{t / \Delta t} \delta X_{\Delta t}[k],
$$

where the process $\left\{\delta X_{\Delta t}[k]\right\}_{k}$ is a noise whose variance is stochastic, i.e.,

$$
\delta X_{\Delta t}[k]=\epsilon_{\Delta t}[k] e^{\omega_{\Delta t}[k]},
$$

where $\omega_{\Delta t}[k]$ is the logarithm of the stochastic variance. More specifically, we will choose $\epsilon_{\Delta t}$ to be a gaussian white noise independent of $\omega$ and of variance $\sigma^{2} \Delta t$. The choice for the process $\omega_{\Delta t}$ introduced in 18], is dictated by the cascade picture. It corresponds to a gaussian stationary process whose covariance can be written

$$
\operatorname{Cov}\left(\omega_{\Delta t}[k], \omega_{\Delta t}[l]\right)=\lambda^{2} \ln \rho_{\Delta t}[|k-l|]
$$


where $\rho_{\Delta t}$ is chosen in order to mimic the correlation structure observed in cascade models with an integral time $T$ :

$$
\rho_{\Delta t}[k]= \begin{cases}\frac{T}{(1 k \mid+1) \Delta t} & \text { for }|k| \leq T / \Delta t-1 \\ 1 & \text { otherwise }\end{cases}
$$

Hereafter, we will refer to the process $\omega(t)$ as the "magnitude process". In order the variance of $X_{\Delta t}(t)$ to converge when $\Delta t \rightarrow 0$, one must choose the mean of the process $\omega_{\Delta t}$ such that 18

$$
E\left(\omega_{\Delta t}[k]\right)=-\operatorname{Var}\left(\omega_{\Delta t}[k]\right)=-\lambda^{2} \ln (T / \Delta t),
$$

for which we find $\operatorname{Var}\left(X_{\Delta t}(t)\right)=\sigma^{2} t$. Let us review the multifractal properties of MRW.

\section{2 $\zeta_{q}$ spectrum: computation of the moments}

The $q$ th-order moment of the increments of the MRW can be computed. Since, by construction, the increments of the model are stationary, the law of $X_{\Delta t}(t+l)-X_{\Delta t}(t)$ does not depend on $t$ and is the same law as $X_{\Delta t}(l)$. In Ref. [18], it is proven that the moments of $X(l) \equiv X_{\Delta t \rightarrow 0^{+}}(l)$ can be expressed as

$$
E\left(X(l)^{2 p}\right)=\frac{\sigma^{2 p}(2 p) !}{2^{p} p !} \int_{0}^{l} d u_{1} \ldots \int_{0}^{l} d u_{p} \prod_{i<j} \rho\left(u_{i}-u_{j}\right)^{4 \lambda^{2}}
$$

where $\rho$ is defined by

$$
\rho(t)=\left\{\begin{array}{ll}
T /|t| & \text { for }|t| \leq T \\
1 & \text { otherwise }
\end{array} .\right.
$$

Using this expression in the above integral, a straightforward scaling argument leads to

$$
M(2 p, l)=K_{2 p}\left(\frac{l}{T}\right)^{p-2 p(p-1) \lambda^{2}},
$$

where we have denoted the prefactor

$$
K_{2 p}=T^{p} \sigma^{2 p}(2 p-1) ! ! \int_{0}^{1} d u_{1} \ldots \int_{0}^{1} d u_{p} \prod_{i<j}\left|u_{i}-u_{j}\right|^{-4 \lambda^{2}} .
$$

Note that $K_{2 p}$ is nothing but the moment of order $2 p$ of the random variable $X(T)$ or equivalently of $\delta_{T} X(t)$. From the above expression, we thus obtain

$$
\zeta_{2 p}=p-2 p(p-1) \lambda^{2}
$$

and by analytical continuation, the corresponding full $\zeta_{q}$ spectrum is thus the parabola

$$
\zeta_{q}=\left(q-q(q-2) \lambda^{2}\right) / 2 .
$$

Let us remark that one can show that $K_{q}=+\infty$ if $\zeta_{q}<0$ (i.e., $q>2+1 / \lambda^{2}$ ) and thus the pdf of $\delta_{l} X(t)$ have fat tails [18]. In order to control the order of the first divergent moment (without changing $\lambda$ ), one could simply choose for the $\epsilon_{\Delta t}$ 's a law with fat tails. Indeed, the prefactor $\sigma^{2 p}(2 p-1)$ !! in Eq. (15) comes directly from the fact that the $\epsilon_{\Delta t}$ 's have been chosen to be Gaussian. Using instead fat tail laws (e.g., t-student laws) would allow us to control the divergence of this prefactor.

In Fig. 3 we have estimated the scaling behavior of the absolute moments $M(q, l)$ for a discrete simulation of a MRW (Fig. 3(a)). In order to simulate the sampling of a time continuous MRW, we have generated a discretized MRW using $\Delta t<<1$ and then subsampled it at the 
sample period 1. Using this procedure, we have generated a $2^{17}$ long time-series using the parameters $\Delta t=1 / 16, T=2^{15}, \sigma^{2}=1$ and $\lambda^{2}=0.03$. In Fig. 2(b), we have plotted, in double logarithmic representation $M(q, l)$ versus $l$ for different values of $q$. In these representations, the linear behavior of each moment indicates that the scaling hypothesis is verified. The estimation of $\zeta_{q}$ (made by estimating the slope of each of such curve) is reported in Fig 2(c). As expected this spectrum is a parabola that is in very good agreement with expression (16).

It is clear that the same power law scaling does not stand when $l$ goes to $+\infty$. Since $\rho(l)=1$ for large $l$ (as compared to $T$ ), we get

$$
\begin{aligned}
& E\left(X(l)^{2 p}\right) \quad \sim_{l>>T} \frac{\sigma^{2 p}(2 p) !}{2^{p} p !} \int_{0}^{l} d u_{1} \ldots \int_{0}^{l} d u_{p} \\
& \sim C l^{p}
\end{aligned}
$$

Thus, there is a cross-over from the parabolic multifractal behavior at time scales $l \leq T$ which is described by Eq. (16) to the Brownian-like behavior at larger time scales $(l>>T)$

$$
\zeta_{q}=q / 2
$$

In Eq. (6), we have shown that there exists a deep link between the self-similarity kernel and the $\zeta_{q}$ spectrum. This suggests that the probability distribution functions of our model satisfy Castaing's equation when going from large to small time scales with a gaussian kernel $G_{l, T}$. Thus, as far as the increment pdf at different time scales are concerned, they will satisfy an evolution equation from "quasi-Gaussian" at very large scale $(l>>T)$ to fat tailed pdf's at small scales. This transformation of the pdf's is illustrated in Fig. 1 (a) where are plotted, in logarithmic scale, the standardized pdf's (the variance has been set to one) for different time scales in the range $[1,4 T]$. The pdf's have been estimated for 500 realizations of size $2^{17}$ of MRW with parameters $\lambda^{2}=0.03$ and $T=2^{13}$. In solid line, we have superimposed the Castaing's transformation obtained from the coarse scale pdf (at scale $T$ ) using the appropriate normal self-similarity kernel. If Fig. 4(b) we have reproduced similar analysis for the S\&P500 future variations. Besides statistical convergence limitations, one can observe the same features as in Fig. 凹(a).

\subsection{Volatility and magnitude correlation functions}

\subsubsection{Volatility correlation functions}

As recalled in the introduction, increments of financial time series are well known to be uncorrelated (for time lags large enough) while their amplitude ("local volatilities") possesses power-law correlations. Let us show that our model satisfies these two properties at all time scales smaller than the "integral time" T. By construction, the increment correlation function,

$$
\left\langle\left(X_{\Delta t}(t+\tau)-X_{\Delta t}(t)\right)\left(X_{\Delta t}\left(t_{1}+\tau_{1}\right)-X_{\Delta t}\left(t_{1}\right)\right)\right\rangle
$$

$\left(\forall\left|t_{1}-t\right|>\tau\right)$, is zero in our model. Let us study the correlation function of the squared increments. Since the increments are stationary, we can choose arbitrarily $t_{1}=0$. Thus we need to compute, in the limit $\Delta t \rightarrow 0$, the following correlation function, that corresponds to a lag $l$ between increments of size $\tau$

$$
C(l, \tau)=\left\langle\left(X_{\Delta t}(l+\tau)-X_{\Delta t}(l)\right)^{2} X_{\Delta t}(\tau)^{2}\right\rangle .
$$

From the results of Ref. [18] and in the case $0 \leq l<T, 0 \leq \tau+l<T$, we get,

$$
C(l, \tau)=\sigma^{4} \int_{l}^{l+\tau} d u \int_{0}^{\tau} d v \rho(u-v)^{4 \lambda^{2}} .
$$



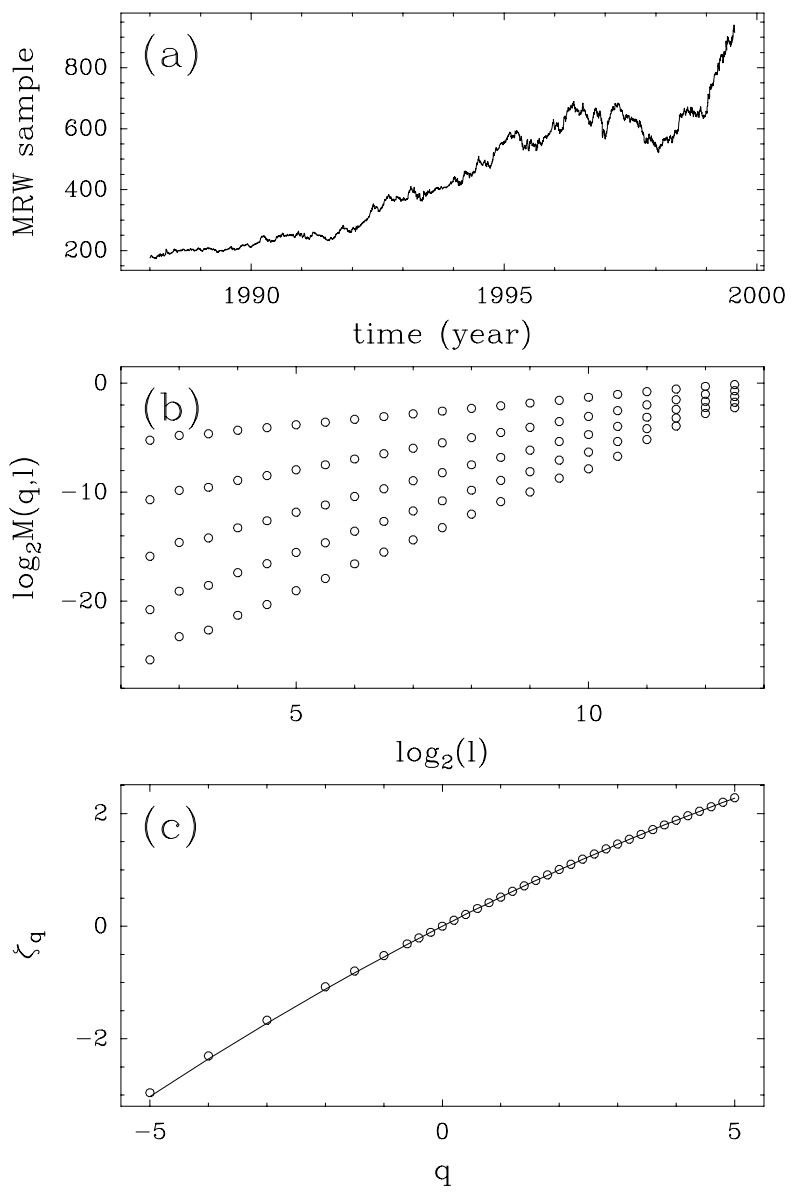

Figure 3: Multifractal Analysis of a MRW sample. (a) Plot of a sample time serie of length $2^{17}$. The sampling size and the trend amplitude have been chosen arbitrarily to be compared to fig 11(a). (b) Log-log plots of $M(q, l)$ versus $l$ for $q=1,2,3,4,5$. The time scales $l$ range from few minutes to one year. (c) $\zeta_{q}$ spectrum estimation (dots) and comparison to prediction as given by Eq. (16) (solid line). 

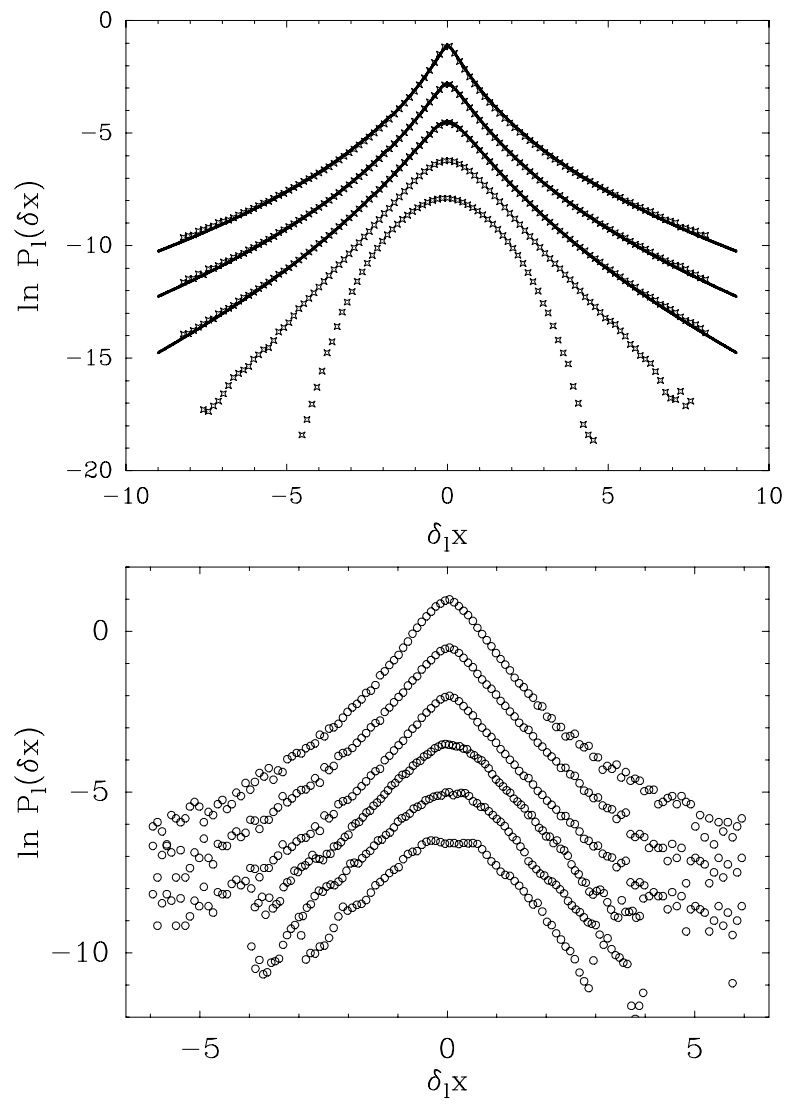

Figure 4: Continuous deformation of increment pdf's across scales. (a) MRW Model. Standardized pdf's (in logarithmic scale) of $\delta_{l} X(t)$ for 5 different time scales (from top to bottom), $l=16,128,2048,8192,32768$. These pdf's have been estimated on 500 MRW realizations of $2^{17}$ sampled points with $\lambda^{2}=0.03, \Delta t=1 / 16$ and $T=8192$. One can see the continuous deformation and the appearance of fat tails when going from large to fine scales. In solid line, we have superimposed the deformation of the large scale pdf using Castaing's equation (5) with the normal self-similarity kernel. This provides an excellent fit of the data. (b) S\&P 500 future. Standardized pdf's at scales (from top to bottom) $l=10,40,160$ min, 1 day, 1 week and one month. As in Fig. (a) the scale is logarithmic and plots have been arbitrarily shifted along vertical axis for illustration purpose. Notwithstanding the small size of the statistical sample (as compared to (a)), one clearly sees the same phenomenon as for the MRW. The fact the Castaing's equation (5) allows one to describe the pdf's deformation across time scales of financial assets has originally been reported in Ref. [9] where similar plots for FX rates can be found. 
A direct computation shows that

$$
\begin{gathered}
\left.\int_{l}^{l+\tau} d u \int_{0}^{\tau} d v \mid u-v\right]^{-4 \lambda^{2}}= \\
\frac{1}{\left(1-4 \lambda^{2}\right)\left(2-4 \lambda^{2}\right)}\left((l+\tau)^{2-4 \lambda^{2}}+(l-\tau)^{2-4 \lambda^{2}}-2 l^{2-4 \lambda^{2}}\right),
\end{gathered}
$$

and consequently

$$
C(l, \tau)=K\left(|l+\tau|^{2-4 \lambda^{2}}+|l-\tau|^{2-4 \lambda^{2}}-2|l|^{2-4 \lambda^{2}}\right)
$$

where

$$
K=\frac{\sigma^{4} T^{4 \lambda^{2}}}{\left(1-4 \lambda^{2}\right)\left(2-4 \lambda^{2}\right)} .
$$

Let us note that in the usual case $0 \leq \tau<<l$, one gets

$$
C(l, \tau) \simeq \sigma^{4} \tau^{2}\left(\frac{l}{T}\right)^{-4 \lambda^{2}}
$$

i.e., for fixed $\tau$, the volatility correlation function scales as

$$
C(l) \sim l^{-2 \nu}
$$

with $\nu=2 \lambda^{2}$. From the estimates $\lambda^{2} \simeq 0.025-0.05$ for financial assets (see section 5), one thus obtains $\nu \simeq 0.05-0.1$, values very close to the ones observed empirically in many works.

\subsubsection{Power of returns and magnitude correlation functions}

Let us now show that magnitude correlation functions behave as expected, i.e, decrease very slowly as a logarithmic behavior.

For that purpose, the previous computation of the correlation function can be extended to the power of returns $\left|X_{\Delta t}(l+\tau)-X_{\Delta t}(\tau)\right|^{p}$. Several empirical works have concerned the study of such "generalized volatilities" and people often noticed variations of amplitude of the correlation and of the power-law exponent $\nu_{p}$ when varying the order $p$ [37]. In Ref. [18], it is shown that the quantity,

$$
C_{p}(l, \tau)=\left\langle\left|X_{\Delta t}(l+\tau)-X_{\Delta t}(l)\right|^{p}\left|X_{\Delta t}(\tau)\right|^{p}\right\rangle,
$$

behaves, when $\tau$ is small enough, as

$$
C_{p}(l, \tau) \sim K_{p}^{2}\left(\frac{\tau}{T}\right)^{2 \zeta_{p}}\left(\frac{l}{T}\right)^{-\lambda^{2} p^{2}}
$$

where the constant $K_{p}$ has been defined previously. Using analytical continuation of the behavior of $C_{p}$ in the limit $p=\epsilon \rightarrow 0$, we can obtain, from previous expression, the behavior of the magnitude correlation function $C_{\omega}(l, \tau)$ :

$$
C_{\omega}(l, \tau) \simeq \epsilon^{-2}\left(C_{\epsilon}(l, \tau)-M(\epsilon, \tau)^{2}\right) \sim-\lambda^{2} \ln \left(\frac{l}{T}\right) .
$$

The magnitude correlation function, for $\tau$ small enough, has thus the same behavior as the

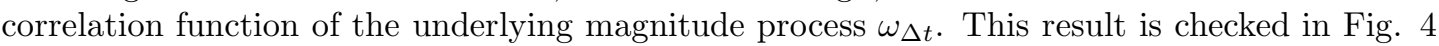
where we have plotted the magnitude correlation function for $\tau=32 \Delta t$ as a function of $\ln (l)$. This correlation function has been estimated using a single realization of the process of $2^{17}$ sampled points, i.e, 16 integral scales. The linear behavior we obtain is exactly the same one as predicted from Eq. (23) and Fig. 2. Measures of the slope and the intercept of such straight line provide a good estimate of respectively $\lambda^{2}$ and $T$. 


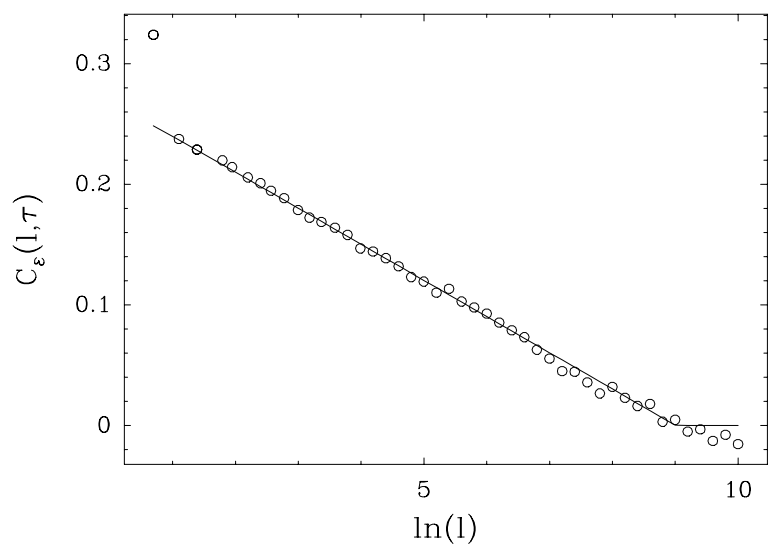

Figure 5: Magnitude correlation function of the model. The correlation function has been estimated on 16 integral scales (see text). In continuous line we have superimposed the correlation function of the magnitude process $\omega_{\Delta t}$ that is involved in the stochastic volatility.

\subsection{Extension to a multivariate process}

In order to account for the fluctuations of financial portfolios and to consider management applications of our approach, it is important to build a multivariate, i.e. a vector valued, version of the previous multifractal random walk. Since only gaussian random variables are involved in the construction of section 4 , this generalization can be done by considering two uncorrelated gaussian random vectors $\epsilon_{\Delta t}(t)$ and $\omega_{\Delta t}(t)$ whose covariance matrices are denoted respectively $\boldsymbol{\Sigma}$ and $\boldsymbol{\Lambda}$. Hereafter, we will refer to these matrices as respectively the "Markowitz matrix" $\boldsymbol{\Sigma}$ and the "multifractal matrix" $\boldsymbol{\Lambda}$. One can then define the multivariate multifractal random walk (MMRW) $\vec{X}(t)$ as:

$$
X_{i}(t+\Delta t)-X_{i}(t)=\epsilon_{i}(t) e^{\omega_{i}(t)}
$$

with $\operatorname{Cov}\left(\epsilon_{i}(t), \epsilon_{j}(t+\tau)\right)=\delta(\tau) \boldsymbol{\Sigma}_{i j}$ and $\operatorname{Cov}\left(\omega_{i}(t), \omega_{j}(t+\tau)\right)=\boldsymbol{\Lambda}_{i j} \ln \left(T_{i j} /|\Delta t+| \tau \mid\right)$ (note that the previously defined coefficients $\sigma^{2}$ and $\lambda^{2}$ for an asset $i$ correspond respectively to the diagonal elements $\boldsymbol{\Sigma}_{i i}$ and $\boldsymbol{\Lambda}_{i i}$ ). Let us briefly review some of the properties of this model, postponing its detailed analysis to a forthcoming publication 17. A quantity that will be of central interest is the $k$-point joint moment of order $q_{1}, q_{2}, \ldots, q_{k}$ that can be defined as:

$$
M_{i_{1}, \ldots, i_{k}}\left(q_{1}, \ldots, q_{k}\right)=E\left(\left|X_{i_{1}}(l)\right|^{q_{1}} \ldots\left|X_{i_{k}}(l)\right|^{q_{k}}\right) .
$$

When $k=2$, by denoting $i_{1}=i, i_{2}=j, q_{1}=p$ and $q_{2}=q$, let us define the joint scaling exponent spectrum as:

$$
M_{i, j}(p, q)=C_{i, j}(p, q) l^{\zeta_{i, j}(p, q)} .
$$

This spectrum can be computed analytically. If the matrix $\boldsymbol{\Sigma}$ is diagonal (the $\epsilon_{i}$ 's are uncorrelated), a straightforward calculation shows that the scaling exponent $\zeta_{i, j}(p, q)$ is the following:

$$
\zeta_{i j}(p, q)=\zeta_{i}(p)+\zeta_{j}(q)-\boldsymbol{\Lambda}_{i j} p q
$$

where $\zeta_{i}(q)$ is the $\zeta_{q}$ spectrum for the component $X_{i}(t)$. Thus, for uncorrelated $\omega_{i}$ 's, one has $\zeta_{i j}(p, q)=\zeta_{i}(p)+\zeta_{j}(q)$ while for the extreme case $\omega_{i}=\omega_{j}$, the exponent becomes $\zeta_{i j}(p, q)=$ $\zeta_{i}(p+q)=\zeta_{j}(p+q)$. The computation of the scaling exponent is trickier for general Markowitz 
and multifractal matrices. Under some mild conditions that are necessary for the existence of a non trivial limit $\Delta t \rightarrow 0^{+}$, one can show that the previous scaling law remains valid even for non diagonal matrix $\boldsymbol{\Sigma}[17$.

In order to define a simple way to get an estimate of the multifractal covariance coefficient $\boldsymbol{\Lambda}_{i j}$, let us define the moment ratio:

$$
R_{i j}(q, l)=\frac{E\left(\left|X_{i}(l)\right|^{q}\left|X_{j}(l)\right|^{q}\right)}{E\left(\left|X_{i}(l)\right|^{q}\right) E\left(\left|X_{j}(l)\right|^{q}\right)} \sim l^{\kappa_{i j}(q)}
$$

From Eq. (27), the value of $\kappa_{i j}(q)$ is simply

$$
\kappa_{i j}(q)=-\boldsymbol{\Lambda}_{i j} q^{2} .
$$

Thus, the non-diagonal element in the multifractal matrix $\boldsymbol{\Lambda}$ corrresponds to the nonlinear behavior of the exponent spectrum $\kappa(q)$ of the moment ratio $R$. Along the same line as for the computation of the magnitude auto-correlation in previous section, one can get the correlation function of magnitudes $\omega_{i}(t, l)$ and $\omega_{j}(t, l)$ from the limit $q \rightarrow 0$ of $R_{i j}(q, l)$ :

$$
\operatorname{Cov}\left(\omega_{i}(t, l), \omega_{j}(t, l)\right) \sim-\boldsymbol{\Lambda}_{i j} \ln (l)+C,
$$

where $C$ is a constant related to $T_{i j}[17$. Thus the scale behavior of the magnitude covariance provides an estimate of the multifractal correlation coefficient $\boldsymbol{\Lambda}_{i j}$. This is the generalization of the classical result in multifractal analysis that relates the intermittency coefficient $\lambda^{2}=\boldsymbol{\Lambda}_{i i}$ to the scale behavior of the variance of the magnitude.

Let us remark that the covariance of the variations of the assets $i$ and $j$ can be obtained by a direct calculation:

$$
\operatorname{Cov}\left(X_{i}(l), X_{j}(l)\right)=\boldsymbol{\Sigma}_{i j} e^{\frac{1}{2}\left(\boldsymbol{\Lambda}_{i i}+\boldsymbol{\Lambda}_{j j}-2 \boldsymbol{\Lambda}_{i j}\right)} l .
$$

This covariance between $X_{i}(t)$ and $X_{j}(t)$ thus depends not only on $\boldsymbol{\Sigma}$, the "Markowitz" covariance matrix, but also on the multifractal matrix $\boldsymbol{\Lambda}$. This expression, allows us to get an estimate of the value of $\boldsymbol{\Sigma}_{i j}$ once the values of $\boldsymbol{\Lambda}$ are known.

Finally, let us mention that the idea of "multivariate multifractality" has been recently introduced in Ref. [19] where the authors propose a phenomenological generalization of Castaing's equation to the multivariate setting. Evidences that financial assets are characterized by non trivial multifractal matrices are also provided. We are currently working to obtain further empirical evidences towards such conclusions. Moreover, a precise link between the present model and the extended Castaing's approach of Ref. [19] is under progress.

\section{Parameter estimation for real financial data}

We have seen that the MRW is characterized mainly by 3 parameters: $\sigma^{2}$, the white noise variance, $T$ the integral scale and $\lambda^{2}$ the magnitude variance. We have shown that this model is able to reproduce all the main features of the future S\&P 500 time series. Natural estimators of those parameters can be defined from the results of previous section. The parameter $\lambda^{2}$ can be obtained from the shape of the $\zeta_{q}$ spectrum that is itself estimated using the scaling behavior of the absolute moments $M(q, l)$. This parameter can also be estimated thanks to the magnitude correlation function $C_{\omega}(l, \tau)$ that behaves as $-\lambda^{2} \ln (l / T)$. From the intercept of such correlation function as a function of $\ln (l)$, we can define an estimator of the integral scale $T$. Finally, the parameter $\sigma^{2}$ can be obtained using the classical relationship $\operatorname{Var}\left(\delta_{l} X_{\Delta t}(t)\right)=\sigma^{2} l$. In this section, we report estimates of the multifractal parameters $\lambda^{2}$ and $T$ for some financial time series. We do not have the ambition to provide fine estimates of those parameters. Our aim is rather to get an idea of realistical values of the parameters of the model for real assets. A 


\begin{tabular}{|c||c|c|c|}
\hline Series & Size & $\lambda^{2}$ & $T$ \\
\hline \hline Future S\&P500 & $7.10^{4}$ & 0.025 & 3 years \\
\hline Future JY/USD & $7.10^{4}$ & 0.02 & 6 months \\
\hline Future Nikkei & $7.10^{4}$ & 0.02 & 6 months \\
\hline Future FTSE100 & $7.10^{4}$ & 0.02 & 1 year \\
\hline S\&P500 index & $6.10^{3}$ & 0.024 & 3 years \\
\hline French index & $6.10^{3}$ & 0.029 & 2 years \\
\hline Italian index & $6.10^{3}$ & 0.029 & 2 years \\
\hline Canadian index & $6.10^{3}$ & 0.024 & 3 years \\
\hline German index & $6.10^{3}$ & 0.027 & 3 years \\
\hline UK index & $6.10^{3}$ & 0.026 & 6 years \\
\hline hong-kong index & $6.10^{3}$ & 0.05 & 3 years \\
\hline
\end{tabular}

Table 1: Multifractal paramater estimates for various assets

precise discussion of the properties of various estimators from a statistical point of view is out of the scope of this paper and will be addressed in a forthcoming publication. Note that similar empirical study has already been performed in Ref. [19]. We have studied some high frequency future time series that are sampled at a 10 min rate over the 7 years period from 1991 to 1997. We have also processed a set of daily index values for 8 different countries over the period from 1973 to 1997. The results are reported in table 1.

We remark that the values of the multifractal parameter $\lambda^{2}$ are all very close to $2.510^{-2}$ (excepted for the hong-kong index). The integral time $T$ values are centered around 3 years but with a large spread. Let us notice that we get an estimate of $\ln (T)$ and thus the error on the estimate of $T$ can be very large. We do not report here the values of the errors and confidence intervals for the proposed estimators that will be studied elsewhere.

\section{Discussion about other approaches and findings}

In this section, we make some comments about related studies that concern multifractals and finance.

\subsection{Turbulence and finance}

The analogy between turbulence and finance has been originally proposed by Ghashghaie et al. [9]. These authors proposed to describe the pdf's of FX price changes at different time scales in the same way physicists describe the pdf's of velocity variations at different space separations in fully developed turbulence. This approach naturally leads to the notions of cascading process, Castaing's formula and multifractality as described in section 2. This work suggests that the key mechanism at the origin of these observations, is an information cascade according to which short-term traders are influenced by long-term traders. This cascade is the analog of the Richardson's kinetic energy cascade in turbulence where small eddies result from the breakdown of larger ones and so on [21]. If the observations reported in Ref. [10] strongly support this point of view, its quantitative understanding in terms of "microscopic" mechanisms remains an open question. In this section we would like to comment about some criticisms that have been raised about the analogy between turbulence and finance. The first one concerns the power spectrum behavior in both situations 11, 12, 21. In turbulence, Kolmogorov theory predicts a $k^{-5 / 3}$ power spectrum that is confirmed in experimental situations. In finance, since 
price fluctuations are almost uncorrelated, they are characterized by a $k^{-2}$ spectrum. For a general multifractal process, the exponent $\beta$ of the power spectrum behavior can be shown to be related [27, 28] to the value of $\zeta_{2}: \beta=1+\zeta_{2}$. Thus, from the cascading process point of view, nothing prevents the exponent $\beta$ from being equal to the exponent of the Brownian motion, i.e., $\beta=2$. In other words, as examplified by the MRW, a cascading process can have uncorrelated increments. We could also remark, that in turbulence $\beta=5 / 3$ has a dimensional origin, i.e., it is the exponent of the spatial spectrum of velocity fluctuations within an Eulerian description. If one adopts a Lagrangian description and one is interested by temporal fluctuations of a fluid particle velocity, then the dimensional value of the power spectrum exponent is $\beta=2$. Thus the value of this exponent is not a pertinent argument to reject the analogy with turbulence. Another difference that has been raised in 2] concerns the behavior of the probability of return to origin $P_{l}(0)$ that has been shown to possess a scaling regime in finance while its behavior is more complex for a turbulent velocity field. First of all, let us point out that whatever the quantity studied (probability of return or absolute moments), it is well known that there is no observed well-defined scaling regime in turbulence: the classical "log-log" plots always display some curvature across scales. This curvature is Reynolds number dependent and several studies suggest that it vanishes, i.e. the field is scale-invariant, only in the limit of infinite Reynolds number [21, 31, 39]. However, within the cascade paradigm and using Castaing's equation, the scaling behavior of the probability of return to origin is easy to show. Indeed, by setting $\delta X=0$ in (5), one obtains, from the definition of $\zeta_{q}$ and the self-similarity kernel:

$$
P_{l}(0)=P_{T}(0) \int G_{l, T}(u) e^{-u} d u=P_{T}(0)\left(\frac{l}{T}\right)^{\zeta_{-1}} .
$$

The exponent for the probability of return to origin is thus simply $\zeta_{-1}$. For the log-normal stochastic volatility model introduced in section 4 , we thus get

$$
P_{l}(0) \sim l^{-\frac{1+3 \lambda^{2}}{2}} .
$$

To conclude, neither the power spectrum exponent, nor the scaling behavior of the probability of return to origin can be used as argument against the existence of a cascading process at the origin of the fluctuations of financial time series.

\subsection{Subordinated processes. Multifractal time}

Subordinated processes are Markov processes in a time variable $\mu(t)$ that is itself an (increasing) random process [30]. Such processes have been introduced in finance by Mandelbrot and Taylor [36] to account for the existence of Levy stable laws as the result of a Brownian motion in some stochastic time. Today, the idea of modelling financial return fluctuations as a Brownian motion in a "fractal time, "trading time" or "financial time" can be found in many approaches. In Refs. 13, 14, the multifractal nature of these fluctuations has been modelled by a (fractional) Brownian motion subordinated with a multifractal stochastic measure. In this section, without any concern for rigor, we would like to make a link between our stochastic volatility approach and the multifractal time approach of Mandelbrot and co-authors. Let us first remark that if we drop the noise $\epsilon$ in Eq. (12) and keep only the stochastic volatility $\sigma(t)$, we can construct a stochastic measure $\mu(d t)$ that satisfies $\mu(d t)=e^{\omega(t)} d t$. Using exactly the same kind of computation as in section 4 , one can show that this measure is stationary and its multifractal spectrum $\tau(q)$ is

$$
\tau(q)=\zeta_{2 q}-1,
$$

as usually defined by

$$
\left\langle\mu([0, t])^{q}\right\rangle \sim t^{\tau(q)+1}
$$


Let us note that the existence and the construction of such a measure that is stationary and possesses a continuous scale invariance, was at the heart of the construction in Refs. 13, 14] and was still an open problem. According to these studies, one can thus construct a multifractal process by simply considering the subordinated process $S(t)=B(\mu([0, t]))$ where $B(t)$ is the standard Brownian motion. The $\zeta_{q}$ spectrum of such process would be exactly the same as the stochastic volatility process defined in section 4 . This is not so surprising since, formally, a differential form for the process $S(t)$ would be $d S=\frac{d \mu}{d t} d B(\mu(t))$. If one assumes that a white noise that is subordinated remains a white noise, one thus obtains $d S=e^{\omega(t)} d B(t)$ that is the equation that defines the MRW of section 4. The questions of well-definiteness of this construction, its statistical properties and the precise mathematical justification of such results, will be addressed in a forthcoming work.

\subsection{Some remarks about Bouchaud, Potters and Meyer's model}

Besides multifractal and cascade pictures, our present approach has been inspired by a recent paper by Bouchaud, Potters and Meyer [38. These authors have proposed a model that is very similar to ours: the stochastic volatility $\sigma(t)$ instead of being log-normal $\left(e^{\omega(t)}\right)$ is a normal $(\omega(t))$ random process with long-range (power-law) correlations. By a simple analytical computation, they have shown that the q-order cumulants of such a process satisfy a simple scaling behavior but the moments display apparent multiscaling caused by a "competition" between the different cumulant behavior on a finite scale range. They thus conclude that a distinction between multifractality and such "apparent multifractality" is a difficult task for finite size time series. As far as multifractal analysis and modelling of financial time series are concerned, this work is very interesting and the previous assertion is undoubtedly difficult to infirm. However, let us remark that in order to illustrate their purpose, Bouchaud et al. choose a "stochastic volatility" $\sigma(t)=$ $e^{\omega(t)}$ instead of their "monofractal" model $\sigma(t)=|\omega(t)|$. The reason invoked by the authors is that the log-normal is "a more realistic time series as compared with real data... without changing the feature of the above model, i.e. the very slow decay of the volatility correlations". They thus claim that the scaling features of both models are the same and thus that the multifractality observed for the simulations of the "log-normal" volatility model is only apparent as predicted by their theory for the "normal" volatility model. The results reported in section 4 can be used to show that this interpretation is not correct. Let us indeed reconsider both results of Ref. [38 and section 4. According to the "normal" volatility model, the moment of order $q=2 p$ is written in terms of cumulants and behaves as [38]:

$$
M(2 p, l=N \Delta t)=A_{2 p, 0} N^{(1-\nu) p}+\ldots+A_{2, ., 2} N^{p}
$$

where the constants $A_{q_{1}, . ., q_{k}}$ depend only $q_{i}, \lambda^{2}$ the variance of $\omega$ and $\nu$ the exponent for the correlation function of $\omega: C_{\omega}(l) \sim l^{-\nu}$. According to this equation, if $N$ is small enough, $M(2 p, l) \sim l^{(1-\nu) p}$ while, for $N$ very large, $M(2 p, l) \sim l^{p}$. The transition scale $N^{*}(q=2 p)$ above which the scaling exponent is $\zeta_{q}=q / 2$ can be estimated if we define it as the scale where the contribution to the moment of order $q$ of the cumulant of order 4 and 2 are equal. Using the expression in Ref. [38] for second and fourth cumulants, $C_{2}$ and $C_{4}$, we can show that at scale $N^{*}(q=2 p)$, we have $(2 p-1) ! ! C_{2}^{p} \simeq p(p-1)(2 p-1) ! ! C_{2}^{p-2} C_{4} / 6$. From the value of $C_{4}$, we obtain $(q>1)$ :

$$
N^{*}(q=2 p)=\left(p(p-1) \nu^{2} 2^{2(\nu-1)} \sum_{m=1}^{+\infty} m^{2(\nu-1)}\right)^{\frac{1}{2 \nu}}
$$

This function only depends on $q$ and $\nu$ and is increasing as $q \rightarrow+\infty$. Thus, the larger the $q$ value, the wider the range of scales on which apparent multifractality exists. However, a numerical computation of the values of $N^{*}$ for $\nu=0.2$ shows that the value $N^{*} \simeq 100$ is reached only 
for the moment of order $p=15$. The greatest moment value attained in practical situations is $q \simeq 6$, for which $N^{*}<1$ ! That means that for all moments less that 10, the model of Bouchaud Potters and Meyer predicts the trivial spectrum $\zeta_{q}=q / 2$ without any cross-over phenomenon. For their numerical simulations, they have used a log-normal model. However, within the lognormal ansatz, the conclusions of Ref. [38] are questionnable since, when $\nu$ is small enough, this model is very close to the model introduced in section 4 . Let us indeed consider as in 38 that $C_{\omega}(l) \sim \lambda^{2}\left(\Gamma(\nu) \cos \left(\frac{\pi \nu}{2}\right)\right)^{\frac{1}{2}} l^{-\nu}$ with $\nu$ very small[ By expanding this expression, we obtain

$$
C_{\omega}(l)=\frac{\lambda^{2}}{\nu}-\lambda^{2} \ln (l)+O(\nu \ln (l)) .
$$

If we set $T=e^{\nu^{-1}}$, then for $1 \leq l<<T$, this equation becomes

$$
C_{\omega}(l)=\lambda^{2} \ln (T / l)
$$

that is the same correlation function as introduced for the multifractal model in section 4 . Let us notice that for $\nu=0.1$ we have $T \approx 2.10^{4}, T \approx 3.10^{5}$ for $\nu=0.08$ and $T \approx 5.10^{8}$ for $\nu=0.05$ !. In this model $T$ is increasing very fast as $\nu$ goes to zero. We can thus conclude, that the model numerically studied in Ref. [38] can be seen as multifractal from one point of view: whatever that scaling range $[1, T]$, there exists $\nu$ small enough $(\nu \approx 1 / \ln (T))$ such that the model displays multiscaling with $\log$-normal $\zeta_{q}$ spectrum in this scale range. According to these remarks, we thus think that the multifractal picture is more realistic to describe multiscaling in financial time series.

\section{Summary and prospects}

In this paper we have reviewed what are the main features of multifractal processes. We have shown that the Multifractal Random Walk is a very attractive alternative to classical cascade processes in the sense that it is stationary, continuously scale-invariant and formulated using a simple stochastic evolution equation. As a model for financial engineering, MRW are interesting for many reasons. First, as illustrated in details for the S\&P 500 intraday time series, this model is able to reproduce the main empirical properties observed for financial time series. Moreover, as Brownian motion and other stable walks, it is a "scale-free" model in the sense that it does not have to fit a particular time-scale since it is scale-invariant. This kind of stability with respect to time "aggregation" is a serious advantage as compared to classical ARCH-like models which parameters strongly depend on the time-scale one is interested in. Moreover, as discussed in section 4.4, a simple multivariate formulation of MRW can be proposed. To our knowledge, it is the first example of an extension of the notions of multifractality to a vector field. The empirical results reported in Ref. [19] suggest that MMRW can be pertinent for portfolio theory. We are currently working on applications of MRW to classical problems of finance like management problems and option pricing theory.

From a theoretical point of view, MRW can be seen as the simplest model that contains the main ingredients for multifractality. In that respect, it can be very helpful to elucidate, in many fields where multiscaling is observed, what are the generic mechanisms that are involved leading to "non-trivial" self-similarity properties. Various "microscopic" models, as proposed in finance or other fields, could be considered within this perspective. It could also be interesting to recast our approach within a field theoretical formulation involving some renormalization procedure.

\footnotetext{
${ }^{7}$ Notice that there is no reason to consider the same value of $\nu$ for the normal and log-normal models. The results of this paper suggest that, in finance, the value for the correlation exponent in the log-normal model is very close to zero and significantly smaller than 0.2
} 
From a mathematical point of view, this problem is deeply linked to the existence of a limit stochastic process when the sampling time $\Delta t$ goes to zero. The convergence of the moments is not sufficient to prove this non trivial assertion. Such a limit could be very useful to develop a new stochastic calculus within which, for example, one could formulate the model of multifractal time of Mandelbrot and co-authors very naturally (see section 6.2). Finally, in a forthcoming work, we will discuss the generalization of such approach to other laws than the (log-)normal.

\section{Acknowledgement}

We acknowledge Matt Lee and Didier Sornette for the permission to use their financial data. We are also very grateful to Alain Arneodo, Jean-Philippe Bouchaud and Didier Sornette for interesting discussions and remarks.

All the computations in this paper have been made using the free GNU licensed sofware LastWave [10].

\section{References}

[1] J.P. Bouchaud and M. Potters, Theory of financial risk: From statistical physics to risk management (Cambridge University Press, Cambridge, 1999).

[2] R. Mantegna and H.E. Stanley, An introduction to econophysics (Cambridge University Press, Cambridge, 2000).

[3] J.D. Farmer, Computing in Science \& Engineering, Nov. 1999, 26. and references therein.

[4] B.B. Mandelbrot, J. Business, 36 1963, 394.

[5] E.F. Fama, J. Business, 38 1965, 34.

[6] R.F. Engle,Econometrica, 50 1982, 987.

[7] T. Bollersev, R.Y. Chous and K.F. Kroner, J. Econometrics, 52 1992, 5.

[8] R.N. Mantegna and H.E. Stanley, Nature, 376 1995, 46.

[9] S. Ghashghaie, W. Breymann, J. Peinke, P. Talkner and Y. Dodge, Nature, 381, 1996, 767.

[10] A. Arneodo, J.F. Muzy and D. Sornette, Eur. Phys. J. B, 2 1998, 277

[11] R.N. Mantegna and H.E. Stanley, Nature, 383 1996, 587.

[12] A. Arneodo, J.P. Bouchaud, R. Cont, J.F. Muzy, M. Potters and D. Sornette, Comment on "Turbulent cascades in Foreign exchange markets", preprint cond-mat/9607120 at xxx.lanl.gov .

[13] A. Fisher, L. Calvet and B.B. Mandelbrot, Multifractality of the deutschmark/us dollar exchange rate, Cowles Foundation Discussion Paper, 1997.

[14] B.B. Mandelbrot, Scientific American 280, 70 (1999).

[15] M.E. Brachet, E. Taflin and J.M. Tchéou scaling transformation and probability distributions for financial time series, preprint cond-mat/9905169, 1999.

[16] F. Schmitt, D. Schwertzer and S. Levejoy, Multifractal analysis of Foreign Exchange data, preprint, Mc Gill University, Montreal 1999.

[17] E. Bacry, J. Delour and J.F. Muzy, in preparation.

[18] E. Bacry, J. Delour and J.F. Muzy, A multifractal random walk, Submitted to Phys. Rev. Lett. (April 2000).

[19] J.F. Muzy, D. Sornette, J. Delour and A. Arneodo, Hierarchical portfolio theory and intertemporal stability, preprint (January 2000). 
[20] G.Bonanno, F. Lillo and R. Mantegna, Dynamics of the number of trades of financial securities, preprint cond-mat 991212006 at xxx.lanl.gov/.

[21] U. Frisch, Turbulence (Cambridge University Press, Cambridge, 1995).

[22] Y. Meyer, Ondelettes (Hermann, Paris, 1990).

[23] I. Daubechies, Ten lectures on wavelet (SIAM, Philadelphia, 1992).

[24] S. Mallat, A wavelet tour in signal processing (Academic Press, New-York, 1998).

[25] J.F. Muzy, E. Bacry and A. Arneodo, Phys. Rev. Lett. 67, 3515 (1991).

[26] E. Bacry, J.F. Muzy and A. Arneodo, J. Stat. Phys. 70, 635 (1993).

[27] J.F. Muzy, E. Bacry and A. Arneodo, Int. J. of Bif. and Chaos 4, 245 (1994).

[28] A. Arneodo, E. Bacry and J.F. Muzy, Physica A 213, 232 (1994).

[29] S. Jaffard, SIAM J. Math. Anal. 28, 944 (1997); SIAM J. Math. Anal. 28, 971 (1997).

[30] W. Feller, An introduction to probability theory and its applications, Vol 2. (John Wiley \& Sons, New-York, 1971).

[31] B. Castaing, Y. Gagne and E. Hopfinger, Physica D 46, 177 (1990); B. Chabaud, A. Naert, J. Peinke, F. Chillà, B. Castaing and B. Hebral, Phys. Rev. Lett. 73, 3227 (1994).

[32] A. Arneodo, S. Roux and J.F. Muzy, J. Phys. II France 7, 363 (1997).

[33] A. Arneodo, E. Bacry and J.F. Muzy, J. Math. Phys. 39, 4163 (1998).

[34] A. Arneodo, E. Bacry, S. Manneville and J.F. Muzy, Phys. Rev. Lett 80, 708 (1998).

[35] M.B. Weissman, Rev. Mod. Physics 60, 537 (1992) and references therein.

[36] B.B. Mandelbrot and H.M. Taylor, Op. Research 15, 1057 (1967).

[37] Z. Ding and C.W.J. Granger, J. of Econometrics 73, 185 (1996).

[38] J.P. Bouchaud, M. Potters and M. Meyer, Eur. Phys. J. B 13, 595 (2000).

[39] A. Arneodo, S. Manneville and J.F. Muzy, Eur. Phys. J. B 1, 129 (1998).

[40] LastWave software freely available at http://wave.cmap.polytechnique.fr/soft/LastWave. 